International Electronic Journal of GeOMEtry

Volume 7 No. 2 PP. 37-46 (2014) (CIEJG

\title{
ADAPTED METRICS AND WEBSTER CURVATURE ON THREE CLASSES OF 3-DIMENSIONAL GEOMETRIES
}

\author{
MIRCEA CRASMAREANU
}

(Communicated by Murat TOSUN)

\begin{abstract}
The Chern-Hamilton notion of adapted metric and the corresponding Webster curvature $W$ is discussed for 3-dimensional unimodular Lie groups, Bianchi-Cartan-Vranceanu metrics and warped metrics. For the first two metrics, we control the value of $W$ by means of two parameters: $A$ and $B$ provided by the Milnor frame in the former case respectively $l$ and $m$ in the latter case. For warped metrics, the value of $W$ depends on the derivatives of the warping function up to order two. Bi-warped 3-metrics are introduced and studied from the point of view of Webster curvature.
\end{abstract}

\section{INTRODUCTION}

Among the Riemannian manifolds of non-constant sectional curvature a special rôle is played by the homogeneous spaces with a large isometry group. Due to the recent approach of Hamilton-Perelman to the Poincaré conjecture (by means of Ricci flow, [8]), a great interest is in dimension 3; for a recent survey on this dimension see [12]. The present note aims to discuss two topics in 3-dimensional Riemannian geometry, both considered by Chern and Hamilton in [5]: adapted metric to a given differential 1-form and the Webster curvature $W$. In fact, we generalize the concept of adapted metric by modifying the original condition of Chern-Hamilton from the scalar 2 to a general $\rho \in \mathbb{R}$ in order to cover all possibilities. The above notions are considered in three settings: unimodular Lie groups, Bianchi-Cartan-Vranceanu geometries and warped metrics.

The Webster scalar curvature $W$ was introduced already into the framework of 3-dimensional geometry of Lie groups by Domenico Perrone [16] in order to classify homogeneous contact metrics on 3-manifolds. More precisely, in the cited paper was proven that a simply connected Riemannian homogeneous contact 3-manifold is a Lie group with a left-invariant contact Riemannian structure and the classification is given in terms of the (non-)unimodularity of the group, its Webster curvature and the torsion invariant $\tau$ introduced also by Chern and Hamilton.

Date: Received: June 25, 2014 and Accepted: September 30, 2014.

2010 Mathematics Subject Classification. 53C30; 53C21; 22E15; 22E30; 53C25; 53C15; 53D35; $53 \mathrm{D} 10$.

Key words and phrases. adapted metric; Webster curvature; unimodular Lie group; Milnor frame; Bianchi-Cartan-Vranceanu metric; warped metric. 
We propose here a new approach for computing $W$ in geometry of a unimodular Lie group $(G, g)$. In fact, we use another formula of [5] which is based on structural equations (see Section 1) through the dual of the Milnor frame ([13]) of $G$. The advantage of our result from Section 3 is that we can control the value of $W$ by means of two parameters, denoted $A$ and $B$ in Section 2, provided by the orthonormality of metric $g$ with respect to Milnor frame. As example of this possibility of control, since $A$ and $B$ are strictly positive, a look at our formula (3.1) yields that the vanishing of $W$ implies that $\lambda$ and $\mu$, the scalars given by Lie brackets of Milnor frame, are of opposite signs.

Regarding the second setting it is well-known that the maximum dimension of the isotropy group of a 3-dimensional manifold is 6 and the fact that there is no metric with 5-dimensional group. The Bianchi-Cartan-Vranceanu spaces are certain 3-dimensional homogeneous Riemannian manifolds with 4-dimensional isometry group. They form a two parameters family containing, among others, some remarkable three-manifolds: $\mathbb{R}^{3}, \mathbb{S}^{3}, \mathbb{S}^{2} \times \mathbb{R}, \mathbb{H}^{2} \times \mathbb{R}$ and the 3-dimensional Heisenberg group $\mathrm{Nil}_{3}$. Recently, several studies are devoted to special submanifolds in these spaces: parallel surfaces [1], biharmonic curves [3] and [6], constant angle surfaces [10], graphs of constant mean curvature [11], biharmonic surfaces [15], higher order parallel and totally umbilical surfaces [17].

Let us pointed out that two motivations for a common presentation of the unimodular Lie groups and Bianchi-Cartan-Vranceanu spaces are provided by:

-there exist remarkable examples belonging to both classes: some of them are listed above,

-in both situations $W$ is controlled by two parameters: in the latter case these are denoted $l$ and $m$ and they have arbitrary signs.

We note that on this way we recover some well-known results but there are also some new computations.

\section{Webster scalar Curvature: the Chern-Hamilton formalism}

Fix $\left(M^{3}, g\right)$ a 3 -dimensional Riemannian manifold and consider $\left\{\omega_{1}, \omega_{2}, \omega_{3}\right\}$ an orthonormal basis of 1 -forms on $M$; let $\left\{e_{1}, e_{2}, e_{3}\right\}$ be the corresponding orthonormal basis of vector fields. There exists a unique skew-symmetric matrix of 1 -forms:

$$
\left(\begin{array}{ccc}
0 & \varphi_{3} & -\varphi_{2} \\
-\varphi_{3} & 0 & \varphi_{1} \\
\varphi_{2} & -\varphi_{1} & 0
\end{array}\right)
$$

such that the structural equations:

$$
\left\{\begin{aligned}
d \omega_{1}=\varphi_{2} & \wedge \omega_{3}-\varphi_{3} \wedge \omega_{2} \\
d \omega_{2}=\varphi_{3} & \wedge \omega_{1}-\varphi_{1} \wedge \omega_{3} \\
d \omega_{3}=\varphi_{1} & \wedge \omega_{2}-\varphi_{2} \wedge \omega_{1}
\end{aligned}\right.
$$

hold on $M$. Making one step further we derive the existence of the functions $\left\{K_{i j} ; 1 \leq i, j \leq 3\right\}$ such that $K_{i j}=K_{j i}$ and:

$$
\left\{\begin{array}{l}
d \varphi_{1}=\varphi_{2} \wedge \varphi_{3}+K_{11} \omega_{2} \wedge \omega_{3}+K_{12} \omega_{3} \wedge \omega_{1}+K_{13} \omega_{1} \wedge \omega_{2} \\
d \varphi_{2}=\varphi_{3} \wedge \varphi_{1}+K_{21} \omega_{2} \wedge \omega_{3}+K_{22} \omega_{3} \wedge \omega_{1}+K_{23} \omega_{1} \wedge \omega_{2} \\
d \varphi_{3}=\varphi_{1} \wedge \varphi_{2}+K_{31} \omega_{2} \wedge \omega_{3}+K_{32} \omega_{3} \wedge \omega_{1}+K_{33} \omega_{1} \wedge \omega_{2} .
\end{array}\right.
$$


Recall that the subject of [5] consists in adapted metrics for a contact 1-form $\omega$ i.e. Riemannian metrics satisfying:

$$
\|\omega\|=1, \quad d \omega=2 * \omega .
$$

If $g$ is adapted to $\omega_{3}$ then the Webster scalar curvature $W$ of the triple $\left(M, g, \omega_{3}\right)$ is defined as:

$$
W\left(M, g, \omega_{3}\right)=\frac{1}{8}\left(K_{11}+K_{22}+2 K_{33}+4\right)
$$

and in the cited paper of Chern and Hamilton is computed for three examples: the unit sphere $\mathbb{S}^{3}$, the unit tangent bundle of a compact orientable surface of genus $g \neq 1$ (for $g=0$ it results $W=1$ ) and the Heisenberg group $\mathrm{Nil}_{3}$. In fact: $W\left(\mathbb{S}^{3}\right)=1$ and $W\left(N i l_{3}\right)=0$.

A second formula on Webster curvature is in $[2$, p. 212] and our relation (4.7) below. Another interpretation of Webster curvature is as the scalar curvature of the Tanaka-Webster connection which is recently studied in arbitrary dimension in [7].

\section{3-DIMENSIONAL UNIMODULAR LIE GROUPS AND ADAPTED METRICS}

Let $G$ be a 3-dimensional Lie group and $\pi: T G \rightarrow G$ its tangent bundle. Suppose that $G$ is unimodular i.e. its volume form is bi-invariant, with a left-invariant metric $g$. Then on $T G$ there exists a left-invariant frame field $\left\{f_{1}, f_{2}, f_{3}\right\}$ with dual co-frame $\left\{\eta^{1}, \eta^{2}, \eta^{3}\right\}$ such that there exist positive constants $A, B, C$ making $g$ a diagonal metric, $[8$, p. 170]:

$$
g=A \eta^{1} \otimes \eta^{1}+B \eta^{2} \otimes \eta^{2}+C \eta^{3} \otimes \eta^{3}
$$

and the Lie brackets are:

$$
\left[f_{i}, f_{j}\right]=c_{i j}^{k} f_{k}
$$

where $c_{i j}^{k} \in\{-2,0,2\}$ and $c_{i j}^{k}=0$ unless $i, j, k$ are distinct. This special frame is usually called Milnor frame. In fact, we work with the associate orthonormal frame:

$$
e_{1}=\frac{f_{1}}{\sqrt{A}}, \quad e_{2}=\frac{f_{2}}{\sqrt{B}}, \quad e_{3}=\frac{f_{3}}{\sqrt{C}}
$$

and denoting $\lambda=c_{23}^{1}, \mu=c_{31}^{2}, \nu=c_{12}^{3}$ we have [8, p. 170]:

$$
\left[e_{i}, e_{j}\right]=\frac{\lambda_{k} c_{i j}^{k}}{\sqrt{\lambda_{1} \lambda_{2} \lambda_{3}}} e_{k}
$$

where $\lambda_{1}=A, \lambda_{2}=B$ and $\lambda_{3}=C$. Let as in previous Section $\left\{\omega^{1}, \omega^{2}, \omega^{3}\right\}$ be the dual co-frame of $\left\{e_{i}\right\}$. More precisely, we have:

$$
\left[e_{1}, e_{2}\right]=\frac{C \nu}{\sqrt{A B C}} e_{3}, \quad\left[e_{2}, e_{3}\right]=\frac{A \lambda}{\sqrt{A B C}} e_{1}, \quad\left[e_{3}, e_{1}\right]=\frac{B \mu}{\sqrt{A B C}} e_{2}
$$

which gives the structural equations:

$$
\left\{\begin{array}{l}
d \omega_{1}=-\frac{A \lambda}{\sqrt{A B C}} \omega_{2} \wedge \omega_{3} \\
d \omega_{2}=-\frac{B \mu}{\sqrt{A B C}} \omega_{3} \wedge \omega_{1} \\
d \omega_{3}=-\frac{C \nu}{\sqrt{A B C}} \omega_{1} \wedge \omega_{2}
\end{array}\right.
$$

and then $g$ is adapted to $\omega_{3}$ if and only if:

$$
\sqrt{C} \nu=-2 \sqrt{A B} \text {. }
$$


In order to enlarge the class of suitable metrics we consider the following notion which appears (with a factor 2 in RHS) in [14]:

Definition 3.1. Fix a 1-form $\omega$ on a general $\left(M^{3}, g\right)$ and the real number $\rho$. The Riemannian metric $g$ on $M$ is called $\rho$-adapted to $\omega$ if $d \omega=\rho * \omega$.

We conclude from (3.6) that:

Proposition 3.1. The metric $g$ is: i) $\frac{-A \lambda}{\sqrt{A B C}}$-adapted to the $\omega_{1}$, ii) $\frac{-B \mu}{\sqrt{A B C}}$-adapted to $\omega_{2}$, iii) $\frac{-C \nu}{\sqrt{A B C}}$-adapted to $\omega_{3}$ in the general case.

Therefore, if the triples $(A, B, C),(\lambda, \mu, \nu)$ are inverse proportional i.e. $A \lambda=$ $B \mu=C \nu=\alpha$ then $g$ is $\frac{-\alpha}{\sqrt{A B C}}$-adapted to all $\omega$ 's. As example we have the 3 -sphere $S^{3}$ where $A=B=C=1$ and $\lambda=\mu=\nu=-2$.

\section{Webster Curvature}

We are ready for the first main result of this note:

Proposition 4.1. If the Riemannian metric $g$ is adapted to $\omega_{3}$ then $\nu=-2$ and $\omega_{3}$ is a contact form with $e_{3}$ its Reeb vector field. The Webster curvature is:

$$
W\left(G, g, \omega_{3}\right)=-\frac{1}{4}\left(\frac{\lambda}{B}+\frac{\mu}{A}\right) .
$$

Proof. From (3.7) it results that $\nu<0$ with the only possible variant $\nu=-2 \neq 0$ and we apply the discussion of [2, p. 223] to conclude the first part. Also, it result:

$$
A B=C \text {. }
$$

Regarding the second part we search for scalars $U, V, W$ such that:

$$
\varphi_{1}=U \omega_{1}, \quad \varphi_{2}=V \omega_{2}, \quad \varphi_{3}=W \omega_{3}
$$

and it results the system:

$$
\left\{\begin{array}{l}
V+W=-\frac{A \lambda}{C} \\
W+U=-\frac{B \mu}{C} \\
U+V=2
\end{array}\right.
$$

The solution is:

$$
\left\{\begin{array}{l}
U=1+\frac{A \lambda-B \mu}{2 C} \\
V=1-\frac{A \lambda-B \mu}{2 C} \\
W=-1-\frac{A \lambda+B \mu}{2 C}
\end{array}\right.
$$

We have also:

$$
\left\{\begin{array}{l}
K_{11}=-\frac{U A \lambda}{C C}-V W \\
K_{22}=-\frac{V B \mu}{C}-W U \\
K_{33}=2 W-U V
\end{array}\right.
$$

which gives:

$$
8 W\left(G, g, \omega_{3}\right)=-\frac{U A \lambda+V B \mu}{C}-2 W+4 W-2 U V+4=-\frac{2}{C}(A \lambda+B \mu)
$$

which gives the final conclusion. 
Example 4.1. I) $N i l_{3}: \lambda=\mu=0, \nu=-2$. We reobtain $W\left(N i l_{3}\right)=0$.

II) $S U(2): \lambda=\mu=\nu=-2$. Then: $W(S U(2), A, B)=\frac{1}{2}\left(\frac{1}{A}+\frac{1}{B}\right)$ and since for the usual metric $A=B=1$ we recast $W\left(S^{3}\right)=1$.

III) $S L(2, \mathbb{R}): \lambda=-2, \mu=+2, \nu=-2$. It results: $W(S L(2, \mathbb{R}), A, B)=$ $\frac{1}{2}\left(\frac{1}{B}-\frac{1}{A}\right)$. Therefore $W(S L(2, \mathbb{R}), 1,1)=0$. The importance of these metrics is connected with Corollary 3.3 of [16, p. 247] that: "the Heisenberg group and the Lie group $\widetilde{S L}(2, \mathbb{R})$ are the only simply connected 3-manifolds which admit an unimodular homogeneous contact Riemannian structure with Webster scalar curvature $W=0 . "$

IV) $E(2)=\operatorname{Isom}\left(\mathbb{E}^{2}\right): \lambda=-2, \mu=0, \nu=-2$. Then: $W(E(2), A, B)=\frac{1}{2 B}>0$. For $B=1$ we reobtain the result of $\left[16\right.$, p. 252] that $W(\widetilde{E}(2))=\frac{1}{2}$.

V) $E(1,1)=S o l: \lambda=+2, \mu=0, \nu=-2$. It follows: $W(S o l, A, B)=-\frac{1}{2 B}<0$.

A second and third formula for the Webster scalar formula holds if the pair $\left(g, e_{3}\right)$ belongs to an almost contact structure [2, p. 213], [16, p. 245], [9, p. 222]:

$$
W\left(M, g, \omega_{3}\right)=\frac{1}{8}\left(r-\operatorname{Ric}\left(e_{3}\right)+4\right)=\frac{1}{8}\left(r+2+\frac{\left\|\tau_{3}\right\|^{2}}{4}\right)
$$

where $r$ is the scalar curvature of the metric $g, \operatorname{Ric}\left(e_{3}\right)$ is the Ricci curvature in the direction of $e_{3}$ and $\tau_{3}=\mathcal{L}_{e_{3}} g$. Note also, that conform [2, p. 214], we have:

$$
r=2 K(\mathcal{D})+2 \operatorname{Ric}\left(e_{3}\right)
$$

where $K(\mathcal{D})$ is the sectional curvature of the 2 -plane $\mathcal{D}=\operatorname{ker} \omega_{3}$.

For our manifold it results, in the general almost contact case:

$$
\left\{\begin{array}{l}
r=-2+\frac{\lambda \mu}{C}+\frac{\mu \nu}{A}+\frac{\nu \lambda}{B}-\frac{1}{2 A B C}\left[A^{2} \lambda^{2}+B^{2} \mu^{2}+C^{2} \nu^{2}\right] \\
\operatorname{Ric}\left(e_{3}\right)=\frac{C^{2} \nu^{2}-(A \lambda-B \mu)^{2}}{2 A B C} \\
K(\mathcal{D})=\frac{(A \lambda-B \mu)^{2}+C \nu(2 A \lambda+2 B \mu-3 C \nu)^{2}}{4 A B C} \\
\left\|\tau_{3}\right\|=2\left[\left|2-\frac{C^{2} \nu^{2}-(A \lambda-B \mu)^{2}}{2 A B C}\right|\right]^{\frac{1}{2}}
\end{array}\right.
$$

In the hypothesis of Proposition 4.1 it results:

$$
\left\{\begin{array}{l}
r=-2-2\left(\frac{\lambda}{B}+\frac{\mu}{A}\right)-\frac{1}{2}\left(\frac{\lambda}{B}-\frac{\mu}{A}\right)^{2} \\
\operatorname{Ric}\left(e_{3}\right)=2-\frac{1}{2}\left(\frac{\lambda}{B}-\frac{\mu}{A}\right)^{2} \\
K(\mathcal{D})=-3-\left(\frac{\lambda}{B}+\frac{\mu}{A}\right)+\frac{1}{4}\left(\frac{\lambda}{B}-\frac{\mu}{A}\right)^{2} \\
\left\|\tau_{3}\right\|=\sqrt{2}\left|\frac{\lambda}{B}-\frac{\mu}{A}\right| .
\end{array}\right.
$$

and, on this way we reobtain (4.1). It results that the adapted metric $g$ is $K$-contact, in fact Sasakian ([4]), if and only if $A \lambda=B \mu$.

\section{Bianchi-Cartan-VRanceanu metrics}

Fix $l$ and $m$ two real numbers and denotes by $M_{m}^{3}$ the manifold $\{(x, y, z) \in$ $\left.\mathbb{R}^{3} ; F(x, y, z)=1+m\left(x^{2}+y^{2}\right)>0\right\}$. We shall consider on $M_{m}^{3}$ the BianchiCartan-Vranceanu metric, [17, p. 343]:

$$
g_{l, m}=\frac{1}{F^{2}} d x^{2}+\frac{1}{F^{2}} d y^{2}+\left(d z+\frac{l y}{2 F} d x-\frac{l x}{2 F} d y\right)^{2} .
$$


An important feature of these metrics is their $S^{1}$-invariance i.e. the invariance with respect to transformations:

$$
\left(\begin{array}{l}
\tilde{x} \\
\tilde{y} \\
\tilde{z}
\end{array}\right)=\left(\begin{array}{ccc}
\cos \varphi & -\sin \varphi & 0 \\
\sin \varphi & \cos \varphi & 0 \\
0 & 0 & 1
\end{array}\right)\left(\begin{array}{l}
x \\
y \\
z
\end{array}\right)
$$

Also, we note the invariance:

$$
g_{-l, m}(x, y, z)=g_{l, m}(-y,-x, z) .
$$

For other remarks concerning these metrics see [17].

An orthonormal basis in $\Omega^{1}\left(M_{m}^{3}\right)$ is:

$$
\omega_{1}=\frac{d x}{F}, \quad \omega_{2}=\frac{d y}{F}, \quad \omega_{3}=d z+\frac{l y}{2 F} d x-\frac{l x}{2 F} d y
$$

and then:

$$
d \omega_{3}=-\frac{l}{F^{2}} d x \wedge d y, \quad * \omega_{3}=\omega_{1} \wedge \omega_{2}=\frac{1}{F^{2}} d x \wedge d y
$$

Since the $g_{l, m}$-dual of $\omega_{3}$ is the vertical vector field $E_{3}=\frac{\partial}{\partial z}$ we may call vertical adapted a metric adapted to $\omega_{3}$. Therefore the only vertical adapted BianchiCartan-Vranceanu metrics are given by $l=-2$; in particular those of the sphere $S^{3}$ and the Heisenberg group $\mathrm{Nil}_{3}$. With respect to the general definition 3.1 the Bianchi-Cartan-Vranceanu metrics are vertical $(-l)$-adapted.

A straightforward computation gives:

$$
\varphi_{1}=-\frac{l}{2 F} d x, \quad \varphi_{2}=-\frac{l}{2 F} d y, \quad \varphi_{3}=\frac{l}{2} d z+\frac{\left(l^{2}-8 m\right) y}{4 F} d x-\frac{\left(l^{2}-8 m\right) x}{4 F} d y .
$$

Also, we derive the matrix of $K$ 's:

$$
\operatorname{diag}\left(\frac{l^{2}}{4}, \frac{l^{2}}{4}, \frac{16 m-3 l^{2}}{4}\right)
$$

which yields the second main result:

Proposition 5.1. The Webster scalar curvature of the triple $\left(M_{m}^{3}, g_{l, m}, \omega_{3}\right)$ is:

$$
W=m+\frac{4-l^{2}}{8}
$$

Remark 5.1. Recall the formula (4.7). The Ricci tensor field of (0,2)-type for Bianchi-Cartan-Vranceanu metrics is computed in [3, p. 124]:

$$
R i c_{11}=R i c_{22}=4 m-\frac{l^{2}}{2}, \quad \operatorname{Ric} c_{33}=\operatorname{Ric}(\xi)=\frac{l^{2}}{2} .
$$

Then an almost contact Bianchi-Cartan-Vranceanu metric has:

$$
r=R i c_{11}+R i c_{22}+R i c_{33}=8 m-\frac{l^{2}}{2}, \quad K(\mathcal{D})=4 m-\frac{3 l^{2}}{4}, \quad\left\|\tau_{3}\right\|=2 \sqrt{2-\frac{l^{2}}{2}}
$$

which yields again (5.8) and implies that $l$ must be considered only in the interval $[-2,2]$. Then the triple $\left(M_{m}^{3}, g_{l, m}, \omega_{3}\right)$ is a Sasakian manifold (i.e. $K$-contact manifold since in dimension 3 these notions coincides) if and only if $l \in\{-2,2\}$. 
Example 5.1. 1) $m=0, l=-2$ is $\mathrm{Nil}_{3}$ and then we recast: $W\left(\mathrm{Nil}_{3}\right)=0$.

2) if $4 m=l^{2}$ (e.q. $\left.m=1, l=-2\right)$ then $\left(M_{3}, g_{l, m}\right)$ is $S^{3}(m) \backslash\{\infty\}$ and then: $W\left(\mathbb{S}^{3}(m)\right)=\frac{m+1}{2}$. We recover: $W\left(S^{3}\right)=1$.

3) $m=0=l$ is the Euclidean $\mathbb{R}^{3}$, thus: $W\left(\mathbb{E}^{3}\right)=\frac{1}{2}$.

4) if $m>0$ and $l=0$ then we have $M_{m}^{3}=\left(S^{2}(4 m) \backslash\{\infty\}\right) \times \mathbb{R}$ and thus: $W\left(S^{2}(m) \times \mathbb{R}\right)=\frac{m}{4}+\frac{1}{2}$.

$5)$ if $m<0$ and $l=0$ then we have $M_{m}^{3}=H^{2}(4 m) \times \mathbb{R}$ where $H^{2}(k)$ is the hyperbolic plane of constant Gaussian curvature $k<0$. Then: $W\left(H^{2}(m) \times \mathbb{R}\right)=$ $\frac{m}{4}+\frac{1}{2}$.

6) if $m>0$ and $l \neq 0$ we get $S U(2) \backslash\{\infty\}$.

$7)$ if $m<0$ and $l \neq 0$ we have $\widetilde{S L}(2, \mathbb{R})$. In conclusion, for $l \in(0,2)$ we get: $W\left(\widetilde{S L}(2, \mathbb{R}), g_{l, \frac{l^{2}-4}{8}}\right)=0$.

Obviously, an important problem is to obtain metrics with prescribed Webster scalar curvature. We conclude with:

Proposition 5.2. Fix $m \in \mathbb{R}$ and let $c \in\left(-\infty, m+\frac{1}{2}\right)$. Then the Bianchi-CartanVranceanu metrics $g_{ \pm l, m}$ with:

$$
l=\sqrt{4+8(m-c)}
$$

have the Webster scalar curvature equal to c. In particular, we can obtain BianchiCartan-Vranceanu metrics with vanishing Webster scalar curvature only for $m \geq$ $-\frac{1}{2}$.

\section{WARPED METRICS}

Let $B$ and $N$ be two smooth manifolds endowed with the Riemannian metrics $g_{B}$ and $g_{N}$ of dimension $b$ and $n$ respectively. Let $f: B \rightarrow \mathbb{R}_{+}^{*}$ be a smooth and strictly positive function. The warped product of $B$ and $F$ with warping function $f$ is the Riemannian manifold:

$$
B \times{ }_{f} N=\left(M_{b+n}, g\right)=\left(B \times N, g_{B}+f^{2} g_{N}\right)
$$

where in the right-hand-side of above equation the function $f$ is in fact $f \circ \pi$ with $\pi: B \times N \rightarrow B$ the projection on the first factor.

In the following we restrict to the case: $B=I$ is an open real interval with the Euclidean metric $g_{B}(z)=d z^{2}$ and $N=\mathbb{E}^{2}$ the Euclidean plane. We use the classical coordinates $(x, y)$ on $\mathbb{E}^{2}$ and $z$ on $I$; therefore the main vector field considered below on $\left(M_{3}, g\right)=I \times_{f} \mathbb{E}^{2}$, namely $\frac{\partial}{\partial z}=\partial_{z}$ will be called the vertical vector field. The warping function is then $f=f(z)$ and for further use we consider the function $F: B \rightarrow \mathbb{R}:$

$$
F=\ln f
$$

Since the warped metric $g$ is:

$$
g=f^{2}(z)\left(d x^{2}+d y^{2}\right)+d z^{2}
$$

we have the orthonormal basis:

$$
\omega_{1}=f(z) d x, \quad \omega_{2}=f(z) d y, \quad \omega_{3}=d z
$$

with the derivatives:

$$
d \omega_{1}=-f^{\prime}(z) d x \wedge d z, \quad d \omega_{2}=-f^{\prime}(z) d y \wedge d z, \quad d \omega_{3}=0
$$


and hence:

$$
\varphi_{1}=f^{\prime}(z) d y, \quad \varphi_{2}=-f^{\prime}(z) d x, \quad \varphi_{3}=0 .
$$

Also, we derive the matrix of $K$ 's:

$$
\operatorname{diag}\left(-\frac{f^{\prime \prime}}{f},-\frac{f^{\prime \prime}}{f},-\left(\frac{f^{\prime}}{f}\right)^{2}\right)
$$

which yields the another main result:

Proposition 6.1. The warped metric (6.3) is not vertically adapted but formally the Webster scalar curvature of the triple $\left(I \times_{f} \mathbb{E}^{2}, g, \omega_{3}\right)$ is:

$$
W\left(\mathbb{E}^{2}, f\right)=\frac{1}{4}\left(2-F^{\prime \prime}(z)-2\left(F^{\prime}(z)\right)^{2}\right) .
$$

Proof. Since $d \omega_{3}=0 \neq 2 * \omega_{3}=2 \omega_{1} \wedge \omega_{2}=2 f^{2} d x \wedge d y$ we have the first part of conclusion; in fact the metric is 0 -adapted to $\omega_{3}$. The matrix (6.7) implies:

$$
W\left(\mathbb{E}^{2}, f\right)=\frac{1}{4}\left[2-\frac{f^{\prime \prime}}{f}-\left(\frac{f^{\prime}}{f}\right)^{2}\right] .
$$

and a straightforward computation of $F^{\prime}$ and $F^{\prime \prime}$ gives the claimed formula (6.8).

As in the previous section we obtain for the almost contact case:

$$
r=-4 \frac{f^{\prime \prime}}{f}-2\left(\frac{f^{\prime}}{f}\right)^{2}, \quad K(\mathcal{D})=-\left(\frac{f^{\prime}}{f}\right)^{2}, \quad\left\|\tau_{3}\right\|=2 \sqrt{2\left(1+\frac{f^{\prime \prime}}{f}\right)}
$$

which yields the necessary condition regarding the warping function:

$$
f^{\prime \prime}(z)+f(z) \geq 0 \text {. }
$$

Example 6.1. For $f(z)=1$ we get the Euclidean $\mathbb{R}^{3}$ and (6.8) yields again: $W\left(\mathbb{E}^{3}\right)=\frac{1}{2}$. The relations $(6.10)$ give:

$$
r=K(\mathcal{D})=0, \quad\left\|\tau_{3}\right\|=2 \sqrt{2}
$$

similar to (5.10) for $m=l=0$ and then the Euclidean 3-geometry with $e_{3}=\frac{\partial}{\partial z}$ is not an almost contact geometry.

Example 6.2. Let $A, B \in \mathbb{R}$ such that the function:

$$
f(z)=A \cos z+B \sin z
$$

is strictly positive on $I$. We have the equality case of $(6.11)$ and then $e_{3}=\frac{\partial}{\partial z}$ is a Killing vector field for the warped metric $g$. The functions $r$ and $K(\mathcal{D})$ are non-constant and $r<-4, K(\mathcal{D})<0$.

Example 6.3. In order to find a constant Webster curvature we have to solve the differential equation:

$$
F^{\prime \prime}+2\left(F^{\prime}\right)^{2}=C=\text { constant }
$$

For $C=0$ we have the 1-parameter family of solutions: $f_{c}(z)=\sqrt{2 z+c}$ for $c$ a real constant. Hence, with $c=0$ and condition (6.11) we obtain $I=\left(\frac{1}{2},+\infty\right)$ and:

$$
W\left(\mathbb{E}^{2}, f(z)=\sqrt{z}\right)=\frac{1}{2}, \quad g=z(d x+d y)^{2}+d z^{2}, \quad r(z)=\frac{1}{2 z^{2}}=-2 K(\mathcal{D})(z) .
$$


For $C=\lambda>0$ we have the 1-parameter family of solutions:

$$
f_{c}(z)=\sqrt{\cosh (\sqrt{2 \lambda} z)+c} .
$$

For $c=0$ the condition $(6.11)$ means $\tanh ^{2}(\sqrt{2 \lambda} z) \leq 2\left(1+\frac{1}{\lambda}\right)$ and since the range of tanh is $(-1,1)$ it follows that $I=\mathbb{R}$ and:

$$
\left\{\begin{array}{l}
W\left(\mathbb{E}^{2}, f(z)=\sqrt{\cosh (\sqrt{2 \lambda} z)}\right)=\frac{2-\lambda}{4}<\frac{1}{2}, g=\cosh (\sqrt{2 \lambda} z)\left(d x^{2}+d y^{2}\right)+d z^{2} \\
r(z)=-4 \lambda+\lambda \tanh ^{2}(\sqrt{2 \lambda} z)<0, \quad K(\mathcal{D})=-\frac{\lambda}{2} \tanh ^{2}(\sqrt{2 \lambda} z)
\end{array} .\right.
$$

For $C=-\lambda<0$ we get again a 1-parameter family of solutions:

$$
f_{c}(z)=\sqrt{\cos (\sqrt{2 \lambda} z)+c} .
$$

For $c=0$ the condition (6.11) means $z \geq \frac{1}{\sqrt{2 \lambda}} \arctan \left(2\left(1-\frac{1}{\lambda}\right)\right)$ and then $\lambda \in(0,1)$. With $I=\left(0, \frac{1}{\sqrt{2 \lambda}} \arctan \left(2\left(1-\frac{1}{\lambda}\right)\right)<\frac{\pi}{2 \sqrt{2 \lambda}}\right)$ we have:

$$
\left\{\begin{array}{l}
W\left(\mathbb{E}^{2}, f(z)=\sqrt{\cos (\sqrt{2 \lambda} z)}\right)=\frac{2+\lambda}{4} \in\left(\frac{1}{2}, \frac{3}{4}\right), g=\cos (\sqrt{2 \lambda} z)\left(d x^{2}+d y^{2}\right)+d z^{2} \\
r(z)=-4 \lambda-3 \lambda \tan ^{2}(\sqrt{2 \lambda} z)<0, \quad K(\mathcal{D})=-\frac{\lambda}{2} \tan ^{2}(\sqrt{2 \lambda} z)
\end{array} .\right.
$$

Let us remark that for all three metrics above we have $K(\mathcal{D})<0$.

In order to enlarge the class of metrics we consider a more general 2-manifold instead of $\mathbb{E}^{2}$ :

$$
g=f^{2}(z)\left(d x^{2}+u^{2}(x) d y^{2}\right)+d z^{2}
$$

which can be called a bi-warped metric. Then the orthonormal basis is:

$$
\omega_{1}=f(z) d x, \quad \omega_{2}=f(z) u(x) d y, \quad \omega_{3}=d z
$$

and hence:

$$
\varphi_{1}=f^{\prime}(z) u(x) d y, \quad \varphi_{2}=-f^{\prime}(z) d x, \quad \varphi_{3}=-u^{\prime}(x) d y .
$$

The matrix of $K$ 's is:

$$
\operatorname{diag}\left(-\frac{f^{\prime \prime}}{f},-\frac{f^{\prime \prime}}{f},-\frac{\left(f^{\prime}\right)^{2} u+u^{\prime \prime}}{f^{2} u}\right)
$$

which yields:

Proposition 6.2. The pair (bi-warped metric (6.20), $\omega_{3}=d z$ ) has the Webster scalar curvature:

$$
W(u, f)=\frac{1}{4}\left(2-\frac{f^{\prime \prime}}{f}-\frac{\left(f^{\prime}\right)^{2} u+u^{\prime \prime}}{f^{2} u}\right)=W\left(\mathbb{E}^{2}, f\right)-\frac{u^{\prime \prime}}{4 f^{2} u} .
$$

Example 6.4. Let us consider the three 2-dimensional geometries of constant curvature:

i) Euclidean: $u(x)=x$. We reobtain (6.8).

ii) Elliptic i.e. $N=S^{2}: u(x)=\sin x$. We have $W\left(S^{2}, f\right)=W\left(\mathbb{E}^{2}, f\right)+\frac{1}{4 f^{2}}$.

iii) Hyperbolic i.e. $N=H^{2}: u(x)=\sinh x$. We derive $W\left(H^{2}, f\right)=W\left(\mathbb{E}^{2}, f\right)-\frac{1}{4 f^{2}}$. 
For the almost contact case we get the same $\left\|\tau_{3}\right\|$ but:

$$
r=-4 \frac{f^{\prime \prime}}{f}-2 \frac{\left(f^{\prime}\right)^{2} u+u^{\prime \prime}}{f^{2} u}, \quad K(\mathcal{D})=-\frac{\left(f^{\prime}\right)^{2} u+u^{\prime \prime}}{f^{2} u}
$$

and it follows:

$$
\begin{cases}r\left(S^{2}, f\right)=r\left(\mathbb{E}^{2}, f\right)+\frac{2}{f^{2}}, & K(\mathcal{D})\left(S^{2}, f\right)=K(\mathcal{D})\left(\mathbb{E}^{2}, f\right)+\frac{1}{f^{2}} \\ r\left(H^{2}, f\right)=r\left(\mathbb{E}^{2}, f\right)-\frac{2}{f^{2}}, & K(\mathcal{D})\left(H^{2}, f\right)=K(\mathcal{D})\left(\mathbb{E}^{2}, f\right)-\frac{1}{f^{2}}\end{cases}
$$

\section{REFERENCES}

[1] Belkhelfa, M., Dillen, F. and Inoguchi, J.-I., Surfaces with parallel second fundamental form in Bianchi-Cartan-Vranceanu spaces, in "PDEs, submanifolds and affine differential geometry" (Warsaw, 2000), 67-87, Banach Center Publ., 57, Polish Acad. Sci., Warsaw, 2002.

[2] Blair, D. E., Riemannian geometry of contact and symplectic manifolds, Second edition. Progress in Mathematics, 203. Birkhäuser Boston, Inc., Boston, MA, 2010.

[3] Caddeo, R., Montaldo, S., Oniciuc, C. and Piu, P., The classification of biharmonic curves of Cartan-Vranceanu 3-dimensional spaces, in "Modern trends in geometry and topology", 121-131, Cluj Univ. Press, Cluj-Napoca, 2006.

[4] Calvaruso, G., Three-dimensional homogeneous almost contact metric structures, J. Geom. Phys., 69(2013), 60-73.

[5] Chern S. S. and Hamilton, R. S., On Riemannian metrics adapted to three-dimensional contact manifolds, With an appendix by Alan Weinstein. Lecture Notes in Math., 1111, Workshop Bonn 1984, 279-308, Springer, Berlin, 1985.

[6] Cho, J. T., Inoguchi J.-I. and Lee, J.-E., On slant curves in Sasakian 3-manifolds, Bull. Austral. Math. Soc., 74(2006), no. 3, 359-367.

[7] Cho, J. T. and Kon, M., The Tanaka-Webster connection and real hypersurfaces in a complex space form, Kodai Math. J., 34(2011), no. 3, 474-484.

[8] Chow, B., Lu, P. and Ni, L., Hamilton's Ricci flow, Graduate Studies in Mathematics, 77, American Mathematical Society, Providence, RI; Science Press, New York, 2006.

[9] Dragomir, S. and Perrone, D., Harmonic vector fields. Variational principles and differential geometry, Amsterdam, Elsevier, 2012.

[10] Fastenakels, J., Munteanu, M. I. and Van Der Veken, J., Constant angle surfaces in the Heisenberg group, Acta Math. Sin. (Engl. Ser.), 27(2011), no. 4, 747-756.

[11] Lee, H., Extensions of the duality between minimal surfaces and maximal surfaces, Geom. Dedicata, 151(2011), 373-386.

[12] McMullen, C. T., The evolution of geometric structures on 3-manifolds, Bull. Amer. Math. Soc., 48(2011), no. 2, 259-274.

[13] Milnor, J., Curvatures of left invariant metrics on Lie groups, Advances in Math., 21(1976), no. 3, 293-329.

[14] Nicolaescu, L. I., Adiabatic limits of the Seiberg-Witten equations on Seifert manifolds, Comm. Anal. Geom., 6(1998), no. 2, 331-392.

[15] Ou, Y.-L. and Wang, Z.-P., Constant mean curvature and totally umbilical biharmonic surfaces in 3-dimensional geometries, J. Geom. Phys., 61(2011), no. 10, 1845-1853.

[16] Perrone, D., Homogeneous contact Riemannian three-manifolds, Illinois J. Math., 42 (1998), no. 2, 243-256.

[17] Van der Veken, J., Higher order parallel surfaces in Bianchi-Cartan-Vranceanu spaces, Results Math., 51(2008) no. 3-4 339-359.

Faculty of Mathematics, 700506, Iaş-ROMANiA

E-mail address: mcrasm@uaic.ro 